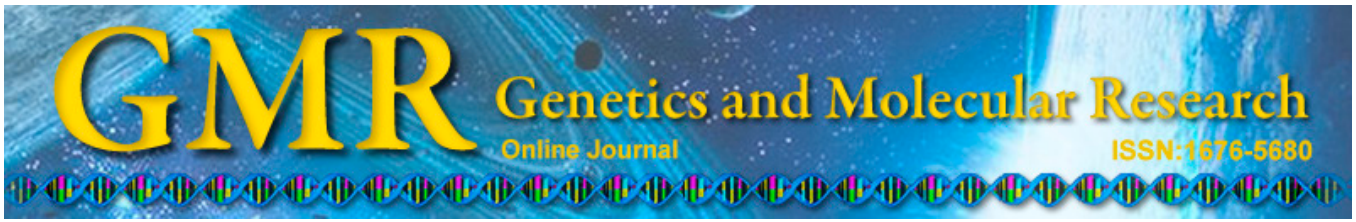

\title{
Effect of GBOT on blood lipid and blood glucose metabolism in rats with atherosclerosis
}

\author{
A. Rong ${ }^{1,2}$, G. Borjihan ${ }^{3}$ and Y.J. Qiao ${ }^{1}$ \\ ${ }^{1}$ School of Chinese Material Medical, Beijing University of Chinese Medicine, \\ Beijing, China \\ ${ }^{2}$ College of Science, Inner Mongolia Agricultural University, Hohhot, China \\ ${ }^{3}$ Institute of Mongolian Medicine Chemistry, Inner Mongolia University, \\ Hohhot, China \\ Corresponding author: Y.J. Qiao \\ E-mail: qiaoyjscmm@126.com
}

Genet. Mol. Res. 14 (3): 7801-7810 (2015)

Received November 3, 2014

Accepted February 5, 2015

Published July 14, 2015

DOI http://dx.doi.org/10.4238/2015.July.14.6

\begin{abstract}
We observed the variation in in vivo blood lipid and blood glucose metabolism in rats with atherosclerosis after 5-(3,4-dihydroxyphenyl)-1-piperidin-1-yl-penta-2,4-dien-1-one (GBOT) administration. Wistar rats aged 10 weeks received a high-fat diet to establish the atherosclerosis model. Metabolic indices related to blood lipid and blood glucose were measured before modeling and at 4 and 8 weeks after modeling. Liver fat levels in rats were measured at 8 weeks to analyze the relationship between liver fat and blood lipid levels. We examined the mechanism of blood lipid reduction. The levels of serum triglycerides, total cholesterol, and very-low-density lipoprotein cholesterol in rats in the control group were significantly decreased $(\mathrm{P}<0.05)$ compared with those in the 4-week control group at 4 weeks and decreased significantly and continuously until the 8 th week $(\mathrm{P}<0.05)$. Compared with the 8-week control group, the blood glucose level in rats in the 8-week experimental group decreased significantly $(\mathrm{P}<0.05)$, and the level of insulin sensitivity
\end{abstract}


index decreased significantly $(\mathrm{P}<0.05)$. Compared with the control group, triglyceride and total cholesterol levels per unit mass in rat liver tissue in the 8 -week experimental group decreased significantly $(\mathrm{P}<0.05)$. Western blotting indicated that GBOT significantly increased the expression of lecithin-cholesterol acyltransferase, low-density lipoprotein receptor, and cholesterol 7 alpha-hydroxylase proteins. GBOT can significantly decrease the levels of blood lipid and blood glucose in rat models of atherosclerosis, and its mechanism may be associated with the promotion of expression of lecithin-cholesterol acyltransferase, low-density lipoprotein receptor, and cholesterol 7 alpha-hydroxylase proteins.

Key words: Atherosclerosis; Blood glucose; Blood lipid metabolism; 5-(3,4-Dihydroxy-phenyl)-1-piperidin-1-yl-penta-2,4-dien-1-one (GBOT)

\section{INTRODUCTION}

Atherosclerosis is the most common and important type of arteriosclerosis and is characterized by lipoid deposition in the intima of the involved artery, accumulation of complex carbohydrates, proliferation of fibrous tissue, calcium deposition, and lesions in the middle layer of the artery (Roy et al., 2012). The disease mainly involves large and middle-sized muscular elastic arteries, including common aortae, coronary arteries, and cerebral arteries, and often leads to a series of severe consequences, including lumen occlusion and wall rupture hemorrhage (Thej et al., 2012; Vukovic et al., 2012). GBOT, 5-(3,4-dihydroxy-phenyl)-1-piperidin-1-yl-penta-2,4dien-1-one, is a derivative of piperine (Figure 1) and cinnamamide alkaloid. It exists extensively in nature, particularly in the Piperaceae plants (Venkatasamy et al., 2004). The pharmacological effects of piperine and its derivatives are extensive, including oxidation resistance, immunoregulation, antineoplastic, and drug metabolism promotion activities, among others (Tu et al., 2014). They can be used to treat central nervous system diseases and have received increasing attention in studies in recent years (Mao et al., 2014). Fructus piperis longi, also known as Zandan-11 in Mongolian medicine, is known to play a role in reducing blood fat. The Mongolian medicine, Gegenqin, containing piperine has been used to treat hyperlipidemia and achieved moderate therapeutic effect (Feng et al., 2014). Animal experiments have also demonstrated that piperine not only reduces serum total cholesterol (TC) in rats with hyperlipidemia, but also prevents the formation of atherosclerosis in domestic rabbits with experimental hypercholesteremia (Bojjireddy et al., 2014). Experimental results have also indicated that piperine can reduce the levels of serum TC, low-density lipoprotein cholesterol, and apolipoprotein B in domestic rabbits with experimental hypercholesteremia and has no significant effect on triglyceride (TG) levels.

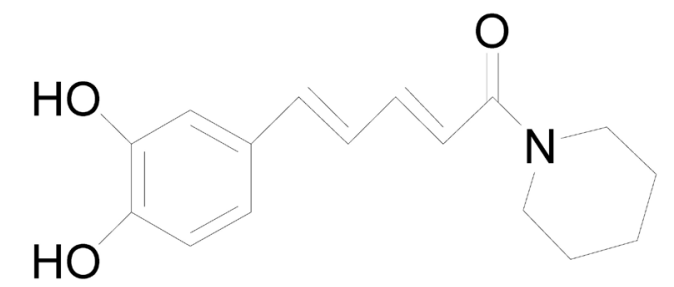

Figure 1. Chemical structural formula of 5-(3,4-dihydroxy-phenyl)-1-piperidin-1-yl-penta-2,4-dien-1-one (GBOT). 
This study provides a theoretical foundation for determining the clinical effect of GOBT on blood lipid and blood glucose in rats with atherosclerosis and for preliminary examination of its mechanism in reducing blood lipid.

\section{MATERIAL AND METHODS}

\section{Experimental animals}

We selected 50 male Wistar rats aged 10 weeks, weighing 200-220 g, Class SPF (Laboratory Animal Centre of Inner Mongolia University). The animals were raised in a room with a constant temperature and humidity and exposed to sunlight for $12 \mathrm{~h}$ each day. The animals were adapted to the environment for 1 week before the experiment. The animals were fed ordinary rodent animal feed and given ad libitum access to food and water.

\section{Establishment of atherosclerosis models}

The experimental rats were administered intraperitoneal injection of vitamin D3 at a dose of $700 \mathrm{kU} / \mathrm{kg}$ for 3 consecutive days and fed $20 \mathrm{~g}$ high-fat diet, which contained $3 \%$ cholesterol, $0.5 \%$ sodium cholate, $0.2 \%$ propylthiouracil, $5 \%$ white sugar, $10 \%$ lard, and $81.3 \%$ basal feed. The rats were raised for 21 consecutive days for successful establishment of the models (Lin et al., 2012; Matsui et al., 2012).

The rats were randomly divided into 4 groups (control group, high-fat group, simvastatin group, and $20 \mathrm{mg} / \mathrm{kg}$ GBOT group) with 10 rats in each group after successful establishment of the models. The rats in the experimental group were administered intraperitoneal injection of 20 $\mathrm{mg} / \mathrm{kg}$ GBOT each day. The rats in the blank control group were administered intraperitoneal injection of $10 \mathrm{~mL}$ normal saline. All experimental rats received treatment for 8 consecutive weeks.

\section{Determination of index levels related to body weight, blood glucose, and blood lipid metabolism}

The blood glucose and blood lipid levels in the 6 animals were determined at 0 weeks and at 4 and 8 weeks after modeling and before euthanization, respectively. The rats were weighed after being fasted for $10 \mathrm{~h}$. Next, $2-3 \mathrm{~mL}$ blood was drawn via the angular vein after the rats received an intraperitoneal injection of $10 \%$ chloral hydrate solution for anesthetization. The fresh blood was placed in a test tube and centrifuged at $1450 \mathrm{~g}$ for $15 \mathrm{~min}$. The serum was pipetted, aliquoted, and cryopreserved at $-80^{\circ} \mathrm{C}$ for detection of the levels of fasting blood glucose and blood insulin. Additional partial serum was collected to detect the levels of indices such as TG, TC, high-density lipoprotein cholesterol, and low-density lipoprotein cholesterol (BioSino Bio-Technology and Science, Inc., Beijing, China).

\section{Extraction and quantification of lipids in rat livers}

The lipids in the rat liver tissue were extracted using Freund's method (including TG and TC). The specific method was used as previously described (Magrini et al., 2013). The levels of TG and TC were determined using reagent kits (Shanghai Yanjing Biotechnology Co., Ltd., Shanghai, China). 


\section{Western blot detection}

The liver tissues of rats were subjected to lysis and sodium dodecyl sulfate-polyacrylamide gel electrophoresis. The wet transfer method was used for $2 \mathrm{~h}$ after electrophoresis at a constant voltage of $120 \mathrm{~V}$. Proteins were electrically transferred to a polyvinylidene fluoride membrane, placed in a Tris-buffered saline containing Tween 20-blocking solution including $5 \%$ skim milk for $1 \mathrm{~h}$ at room temperature, and washed with the same buffer without skim milk before the primary antibody was added. The membrane was shaken at $4{ }^{\circ} \mathrm{C}$ overnight and then washed with Tris-buffered saline containing Tween 20 several times. Rabbit anti-humanrelated protein polyclonal antibodies were added (Santa Cruz Biotechnology Inc., Santa Cruz, CA, USA). The membrane was washed thrice after shaken for $1 \mathrm{~h}$ at room temperature. It was subjected to electrochemiluminescence color development to detect the levels of lecithincholesterol acyltransferase (LCAT), low-density lipoprotein receptor (LDLR), and cholesterol 7 alpha-hydroxylase proteins (CYP7A1).

\section{Statistical analysis}

The SPSS 15.0 software was used for statistical data analysis (SPSS, Inc., Chicago, IL, USA). The continuous variable was consistent with normally distributed measurement data and is reported as means \pm standard deviation. Data were subjected to an independent sample $t$-test or a paired $t$-test. $\mathrm{P}<0.05$ indicated that the difference was statistically significant.

\section{RESULTS}

\section{General observation}

The appearance of the aorta of rats in the experimental group was initially similar to that in the control group. The aorta showed uneven thickness, significantly thickened and hardened vascular walls, and an alternate yellow-and-white color. Significant thickening of the vascular walls and raised yellowish white streaks or yellowish white plaques of different sizes toward the vascular lumen were observed with naked eye. The aorta gradually regained its original elasticity, had an even pink color, and a smooth and bright intima 4 and 8 weeks after administration of GBOT.

Hematoxylin and eosin staining showed that the endothelial cells of the aorta were complete, the monolayer clung to the internal elastic lamina, and the smooth muscle cells were oval and arranged in order in the middle layer. Plaques were formed in most regions of the aortae in the control group, the plaque surface was covered with fibrous tissue, the intima and medial membrane had significantly thickened, the endothelium was partially detached and incomplete, lipid infiltration, substantial foam cells, and inflammatory cell infiltration were present in the endothelium, the smooth muscle proliferated and migrated towards the intima, the elastic lamina was discontinuous, and the elastic fibers were fractured and dissolved (Figure 2).

\section{Changes in body weight of rats in the experimental group}

The body weight of rats in the experimental group increased slowly after administra- 
tion of GBOT. There was a significant difference compared with that at 4 and 8 weeks $(\mathrm{P}<$ 0.05 ; Figure 3).
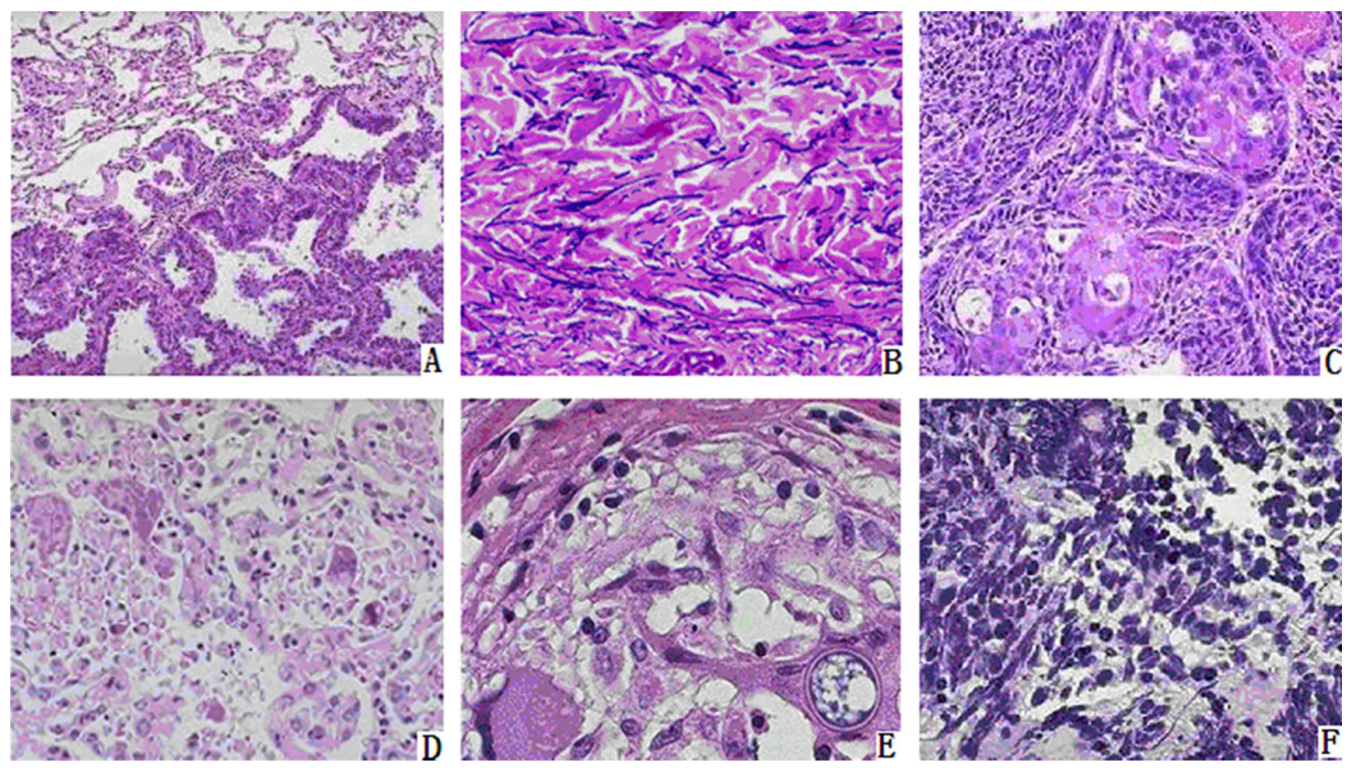

Figure 2. Observation of aorta of rats in the experimental group and control group at different points in time (200X) after HE staining. A. At the time of successful establishment of models in the control group. B. At 4 weeks in the control group. C. At 8 weeks in the control group. D. At the time of successful establishment of models in the experimental group. E. At 4 weeks in the experimental group. F. At 8 weeks in the experimental group.

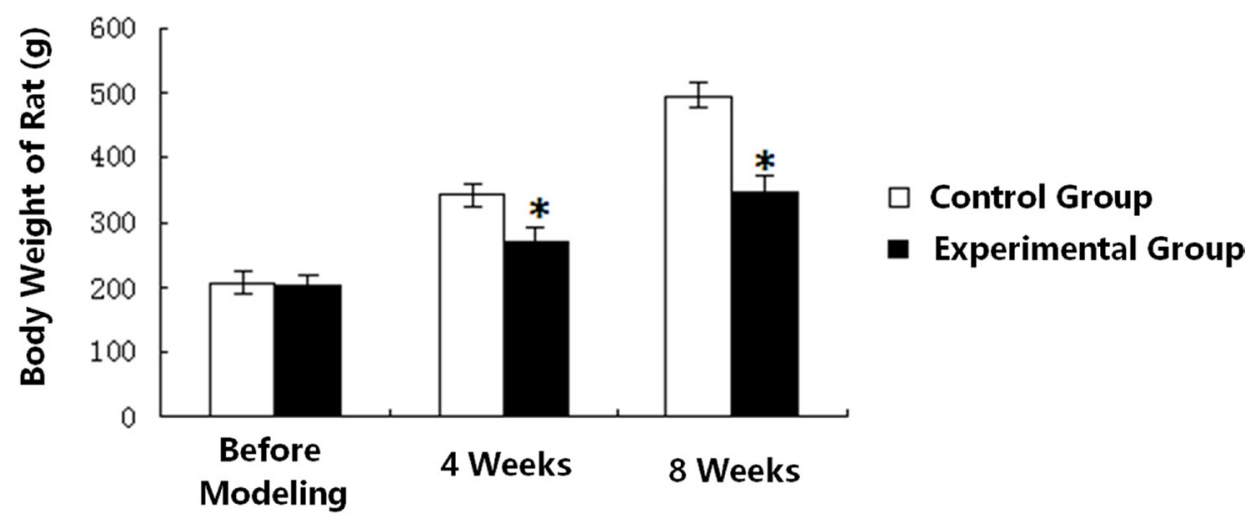

Figure 3. Changes in body weight of rats. $* \mathrm{P}<0.05$ compared with the control group.

\section{Changes in level of blood lipids in experimental rats}

The levels of serum TG, TC, and very-low-density lipoprotein cholesterol in rats in the control group significantly decreased compared with those in the 4-week control group at 4 weeks and decreased significantly and continuously until the 8th week $(\mathrm{P}<0.05)$. 
Before establishing the experimental group, there was no significant statistical difference in serum high-density lipoprotein cholesterol and low-density lipoprotein cholesterol at 4 and 8 weeks between the rats in the experimental group and control group $(\mathrm{P}>0.05$; Table 1$)$.

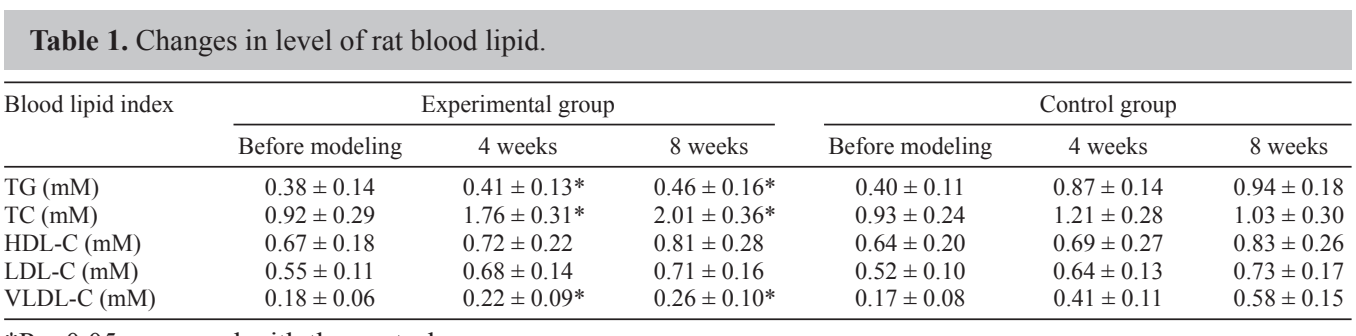

${ }^{*} \mathrm{P}<0.05$ compared with the control group.

\section{Changes in indices related to blood glucose insulin in experimental rats}

In terms of glycometabolism, the baseline value levels of blood glucose insulin indices were the same between the 2 groups of animals. Compared with the 8-week control group, blood glucose level of the rats in the 8-week experimental group decreased significantly $(\mathrm{P}<$ $0.05)$, and the insulin sensitivity index (ISI) decreased significantly $(\mathrm{P}<0.05)$, but the increase in ISI was not significant $(\mathrm{P}>0.05$; Table 2$)$.

Table 2. Changes in indices related to blood glucose insulin in rats.

\begin{tabular}{lccccccr}
\hline Blood lipid index & \multicolumn{3}{c}{ Experimental group } & & \multicolumn{3}{c}{ Control group } \\
\cline { 2 - 3 } & Before modeling & 4 weeks & 8 weeks & & Before modeling & 4 weeks & 8 weeks \\
\hline FBG (mM) & $7.92 \pm 0.24$ & $8.71 \pm 0.28^{*}$ & $8.23 \pm 0.36^{*}$ & & $8.02 \pm 0.30$ & $12.67 \pm 0.45$ & $16.52 \pm 0.68$ \\
INS (U/L) & $11.83 \pm 1.25$ & $12.91 \pm 1.62$ & $13.88 \pm 1.96$ & & $11.79 \pm 1.36$ & $13.52 \pm 1.84$ & $14.59 \pm 2.07$ \\
ISI index & $-3.65 \pm 0.25$ & $-3.79 \pm 0.31^{*}$ & $-3.95 \pm 0.34^{*}$ & & $-3.72 \pm 0.29$ & $-5.81 \pm 0.47$ & $-6.88 \pm 0.59$ \\
\hline
\end{tabular}

$* \mathrm{P}<0.05$ compared with the control group.

\section{Changes in content of lipids in hepatic tissues in experimental rats}

The results indicated that the levels of TG and TC per unit mass in the liver tissue of rats in the 8 -week experimental group decreased significantly $(\mathrm{P}<0.05)$ compared with the control group (Figure 4).

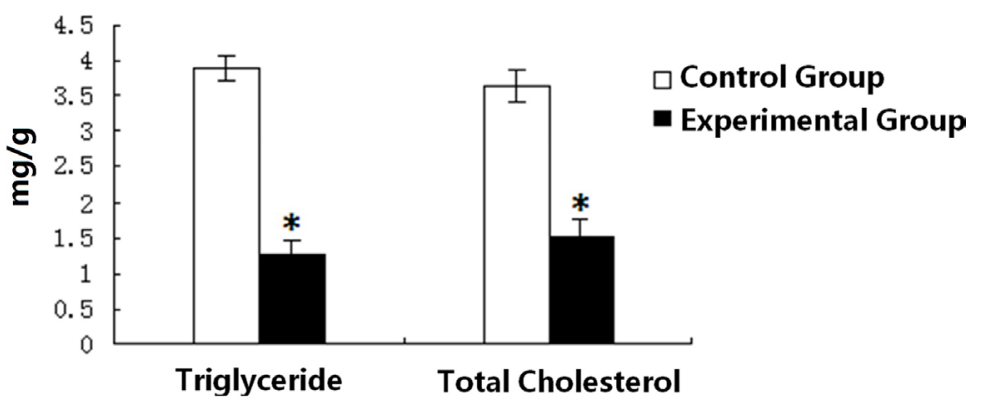

Figure 4. Comparison of levels of triglyceride and total cholesterol in hepatic tissue 8 weeks after modeling between the 2 groups. ${ }^{*} \mathrm{P}<0.05$ compared with the control group. 


\section{Western blotting}

The effect of the piperine derivative GBOT on the expression of proteins related to blood lipid metabolism in rats was evaluated based on 3 proteins. The 3 proteins, that is, hepatic LDLR, CYP7A1, and LCAT were detected using Western blot analysis. The results of protein expression were essentially consistent based on the reverse transcription-polymerase chain reaction results; the polymerase chain reaction result was discussed in another paper (Bao et al., 2012). Hepatic lecithin-cholesterol acyltransferase protein expression is shown in Figure 5.
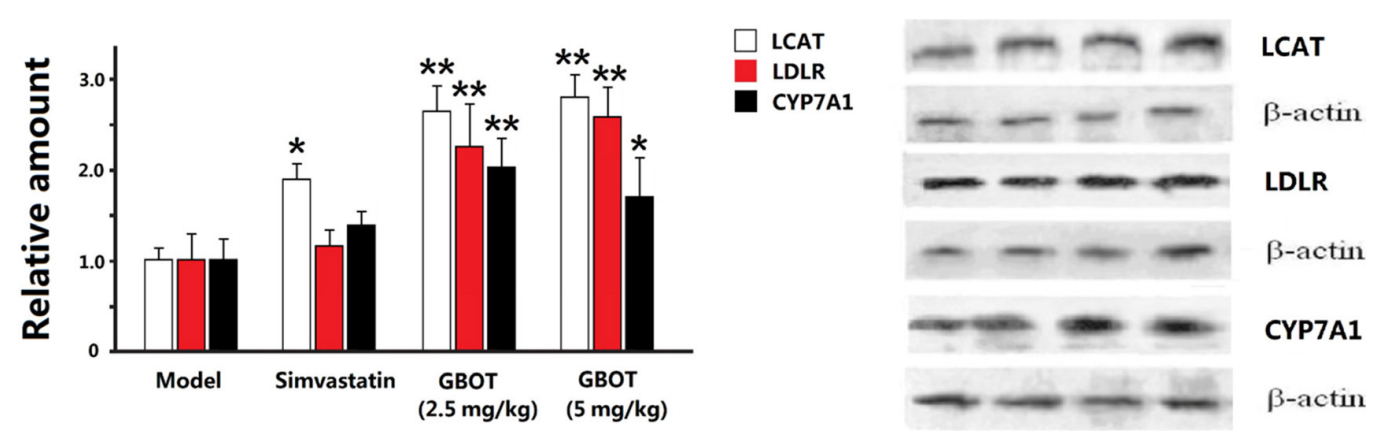

Figure 5. Relative amount of the Western blots of lecithin-cholesterol acyltransferase (LCAT), low-density lipoprotein receptor (LDLR), and cholesterol 7 alpha-hydroxylase proteins (CYP7A1) expression on hyperlipidemia rats (means $\pm \mathrm{SE}, \mathrm{N}=3$ ). $* \mathrm{P}<0.05$ and $* * \mathrm{P}<0.01$, compared with the model group.

\section{DISCUSSION}

Atherosclerosis is a chronic arterial disease that may trigger coronary heart disease and cerebral infarction. Slow progress has been made in understanding the causes and pathogenesis of atherosclerosis. This is largely because of the complex causes of atherosclerosis, slow progression of the disease, and lack of symptoms in the early stage (Akyildiz et al., 2013). Thus, somatic data obtained in most previous studies are mostly cross-sectional, and it is difficult to determine their causal relationship; atherosclerosis lesions in many animal models develop quickly. In recent years, rabbit models of hereditary hyperlipidemia have been developed, but observing subtle and important changes during natural formation of lesions similar to humans over several years in animal models remains challenging (Liu et al., 2014). To study the effect of GBOT on blood lipid and blood glucose metabolism and define the relationship between GBOT and changes in indices related to blood lipid and blood glucose of rats with atherosclerosis we established rat models of atherosclerosis.

To study the antioxidant activity of natural piperine, some previous studies examined the effect of factors such as light, heat, and $\mathrm{pH}$ on piperine. The results showed that piperine have antioxidant activity; their antioxidant ability is equivalent to $60.05 \%$ of vitamin $\mathrm{E}$, and their scavenging activity increases with increasing concentration, but the inhibition ratio decreases and then stabilizes when the mass concentration reaches $4 \mathrm{~g} / \mathrm{L}$ (Bukhari et al., 2013). Piperine derivatives are stable after insulation treatment for $2 \mathrm{~h}$ at $60^{\circ} \mathrm{C}$, but irradiation with natural light can lead to decreased absorbance (Shenoy et al., 2014). The male rats were di- 
vided into 8 groups. The rats in the 4 groups were allowed to fast and were given $1 \%$ carboxymethyl cellulose, $10 \mathrm{mg} / \mathrm{kg}$ body weight carbimazole, $10 \mathrm{~g} / \mathrm{kg}$ carbimazole $+40 \mathrm{mg} / \mathrm{kg}$ body weight piperine, and $10 \mathrm{mg} / \mathrm{kg}$ carbimazole $+2 \mathrm{mg} / \mathrm{kg}$ body weight atorvastatin. The rats in the other 4 groups were subjected to the same treatment except they were given a high-fat diet rather than fasting. Kumar et al. (2013) have examined erythrocyte osmotic fragility, TC, phospholipid, lipid peroxide, superoxide dismutase, catalase, glutathione peroxidase, vitamin E, reduced glutathione, and serum vitamin $\mathrm{C}$, among other properties. Piperine can significantly protect erythrocytes in rats with hyperlipidemia and has significant antioxidant effects.

It has been demonstrated that free radical injury and lipid peroxidation are the leading causes of atherosclerosis, cancers, hepatopathy, and caducity (Lin et al., 2014). GBOT can inhibit the peroxidation products induced by the carcinogenic substances in the intestinal mucosa and increase the activity of glutathione (GSH) and Na-K-ATP enzyme. Antioxidation of GBOT adjusts oxidation changes by inhibiting lipid peroxidation and indirectly increasing synthesis and conduction of GSH (Ouyang et al., 2013). Oxidative stress models of rats fed high-fat diet received GBOT. The levels of all the three GBOT-treated groups were similar to the levels observed in the normal group (Umar et al., 2013). The in vitro test showed that GBOT is free-radical scavenger at a low concentration, but the number of hydroxide radicals increased at a high concentration. GBOT is a strong superoxide scavenger when its $50 \%$ minimum inhibitory concentration is $1.82 \mathrm{mM}$ (Sehgal et al., 2013). In a PC12 cell model, GBOT significantly reduced 1-methyl-4-phenylpyridinium-induced nuclear damage, changes in mitochondrial membrane permeability, reactive oxygen formation, and GSH exhaustion. Its effect is dose-dependent (Ying et al., 2013). The synthetic piperine derivative, piperonyl carboxylic ester, was used to examine blood fat reduction in experimental animals. The derivative, with its molecular structure containing 1 piperonyl, plays a significant role in reducing serum cholesterol, but its role in reducing TGs is not significant; the derivative containing 2 radicals plays a significant role in reducing cholesterol and TG, but its role in reducing cholesterol is less significant than the role of the derivative containing only 1 piperonyl (Wang et al., 2013a).

In this study, the metabolic disturbance of the blood lipid profile dominated by a rise in serum TG occurred, and TG continuously and significantly increased at 4 weeks after establishment in experimental group rat models. The result was similar to the decreased serum TG in rats with atherosclerosis as observed by Choi et al. (2013). Hypertriglyceridemia is an independent risk factor of coronary heart disease, but its mechanism in causing atherosclerosis remains unclear. A recent study showed that TG-rich lipoprotein (TRL) and its TRL remnants might play an important role in formation of atherosclerosis (Meghwal and Goswami, 2013). TRL is synthesized by the liver or generated through absorption of fat by the small intestines. It is secreted into the blood in the form of chylomicrons rich in TG and very-low-density lipoprotein rich in TG (Nirmal et al., 2013). In blood vessels, TRL is subjected to intravascular lipolysis through the activities of enzymes such as lipoprotein lipase, resulting in the formation of the TRL residue. The TRL residue lacks TG, but is rich in cholesterol ester and apolipoprotein E. It has a small volume, higher density, and higher level of cholesterol than TRL. The level of very-low-density lipoprotein cholesterol increases in the blood (Banji et al., 2013). The TRL residue is an important part of small and dense low-density lipoprotein, and the latter has a closer relationship with atherosclerosis than low-density lipoprotein cholesterol level (Wang et al., 2013b).

In conclusion, the piperine derivative GBOT enabled lipid metabolism in rats to regu- 
late lipid metabolism disorders. These compounds can improve the transport lipids and allow for the lipolysis of free fatty acid through many approaches; the $10 \mathrm{mg} / \mathrm{kg}$ GBOT group had the best effect in reducing blood fat. Administration of the piperine derivative can significantly reduce the blood lipid level in animals. The piperine derivative may increase the expression level of LCAT and CYP7A1 and thus affect the increase in LDLR (Konturek et al., 2007).

\section{REFERENCES}

Akyildiz HY, Karabacak A, Akyüz M, Sözüer E, et al. (2013). Effects of piperine in experimental intestinal ischemia reperfusion model in rats. Ulus Travma Acil Cerrahi Derg 19: 387-391.

Banji D, Banji OJ, Dasaroju S and Kranthi KC (2013). Curcumin and piperine abrogate lipid and protein oxidation induced by D-galactose in rat brain. Brain Res. 15: 1-11.

Bao L, Bai S and Borijihan G (2012). Hypolipidemic effects of a new piperine derivative GB-N from Piper longum in high-fat diet-fed rats. Pharm. Biol. 50: 962-967.

Bojjireddy N, Sinha RK and Subrahmanyam G (2014). Piperine inhibits type II phosphatidylinositol 4-kinases: a key component in phosphoinositides turnover. Mol. Cell. Biochem. 393: 9-15.

Bukhari IA, Pivac N, Alhumayyd MS, Mahesar AL, et al. (2013). The analgesic and anticonvulsant effects of piperine in mice. J. Physiol. Pharmacol. 64: 789-794.

Choi S, Choi Y, Choi Y, Kim S, et al. (2013). Piperine reverses high fat diet-induced hepatic steatosis and insulin resistance in mice. Food Chem. 141: 3627-3635.

Feng X, Liu Y, Wang X and Di X (2014). Effects of piperine on the intestinal permeability and pharmacokinetics of linarin in rats. Molecules 19: 5624-5633.

Konturek PC, Burnat G and Hahn EG (2007). Inhibition of Barret's adenocarcinoma cell growth by simvastatin: involvement of COX-2 and Apoptosis-related proteins. J. Physiol. Pharmacol. 58 (Suppl 3): 141-148.

Kumar S, Sharma S and Vasudeva N (2013). Screening of antidiabetic and antihyperlipidemic potential of oil from Piper longum and piperine with their possible mechanism. Expert Opin. Pharmacother. 14: 1723-1736.

Lin L, Li J, Lv H, Ma Y, et al. (2012). Effect of Lycium ruthenicum anthocyanins on atherosclerosis in mice. Zhongguo Zhong Yao Za Zhi 37: 1460-1466.

Lin Y, Xu J, Liao H, Li L, et al. (2014). Piperine induces apoptosis of lung cancer A549 cells via p53-dependent mitochondrial signaling pathway. Tumour Biol. 35: 3305-3310.

Liu Y, Zhang Y, Lin K, Zhang DX, et al. (2014). Protective effect of piperine on electrophysiology abnormalities of left atrial myocytes induced by hydrogen peroxide in rabbits. Life Sci. 94: 99-105.

Magrini S, Feletti A, Marton E and Longatti P (2013). Gliomas of the pineal region. J. Neurooncol. 115: 103-111.

Mao QQ, Huang Z, Zhong XM, Xian YF, et al. (2014). Piperine reverses the effects of corticosterone on behavior and hippocampal BDNF expression in mice. Neurochem. Int. 74: 36-41.

Matsui T, Oka M, Fukui T, Tanaka M, et al. (2012). Suppression of bladder overactivity and oxidative stress by the phytotherapeutic agent, Eviprostat, in a rat model of atherosclerosis-induced chronic bladder ischemia. Int. J. Urol. 19: 669-675.

Meghwal M and Goswami TK (2013). Piper nigrum and piperine: an update. Phytother. Res. 27: 1121-1130.

Nirmal SA, Ingale JM, Pattan SR and Bhawar SB (2013). Amaranthus roxburghianus root extract in combination with piperine as a potential treatment of ulcerative colitis in mice. J. Integr. Med. 11:206-212.

Ouyang DY, Zeng LH, Pan H, Xu LH, et al. (2013). Piperine inhibits the proliferation of human prostate cancer cells via induction of cell cycle arrest and autophagy. Food Chem. Toxicol. 60: 424-430.

Roy SK, Cespedes A, Li D, Choi TY, et al. (2012). Chronic kidney disease is associated with increased coronary artery atherosclerosis as revealed by multidetector computed tomographic angiography. Tex. Heart Inst. J. 39: 811-816.

Sehgal A, Kumar M, Jain M and Dhawan DK (2013). Modulatory effects of curcumin in conjunction with piperine on benzo(a)pyrene-mediated DNA adducts and biotransformation enzymes. Nutr. Cancer 65: 885-890.

Shenoy PA, Nipate SS, Sonpetkar JM, Salvi NC, et al. (2014). Production of high titre antibody response against Russell's viper venom in mice immunized with ethanolic extract of fruits of Piper longum L. (Piperaceae) and piperine. Phytomedicine 21: 159-163.

Thej MJ, Kalyani R and Kiran J (2012). Atherosclerosis in coronary artery and aorta in a semi-urban population by applying modified American Heart Association classification of atherosclerosis: an autopsy study. J. Cardiovasc. Dis. Res. 3: 265-271.

Tu Y, Sun D, Zeng X, Yao N, et al. (2014). Piperine potentiates the hypocholesterolemic effect of curcumin in rats fed on 
a high fat diet. Exp. Ther. Med. 8: 260-266.

Umar S, Golam Sarwar AH, Umar K, Ahmad N, et al. (2013). Piperine ameliorates oxidative stress, inflammation and histological outcome in collagen induced arthritis. Cell Immunol. 284: 51-59.

Venkatasamy R, Faas L, Young AR, Raman A, et al. (2004). Effects of piperine analogues on stimulation of melanocyte proliferation and melanocyte differentiation. Bioorg. Med. Chem. 1905-1920.

Vukovic P, Milojevic P, Peric M and Nezic D (2012). Evaluation of radial artery atherosclerosis by intravascular ultrasound. Angiology 64: 80-81.

Wang J, Vanegas SM, Du X, Noble T, et al. (2013a). Caloric restriction favorably impacts metabolic and immune/ inflammatory profiles in obese mice but curcumin/piperine consumption adds no further benefit. Nutr. Metab. 10: 29-36.

Wang YM, Lin W, Chai SC, Wu J, et al. (2013b). Piperine activates human pregnane X receptor to induce the expression of cytochrome P450 3A4 and multidrug resistance protein 1. Toxicol. Appl. Pharmacol. 272: 96-107.

Ying X, Chen X, Cheng S, Shen Y, et al. (2013). Piperine inhibits IL-beta induced expression of inflammatory mediators in human osteoarthritis chondrocyte. Int. Immunopharmacol. 17: 293-299. 